\title{
Simple Market Equilibria with Rationally Inattentive Consumers
}

\author{
By Filip MatěJKa And Alisdair MCKaY*
}

In some markets, it is difficult for consumers to evaluate the offers made by firms. The literature on consumer search (e.g. Peter A. Diamond, 1971; Kenneth Burdett and Kenneth L. Judd, 1983) has explored the consequences of these information frictions for market equilibria. In this paper we take up the same issue, but with a different way of modeling the information frictions.

Our analysis is built upon the rational inattention framework for modeling information frictions introduced by Christopher A. Sims (2003, 2006). The consumers in our model are able to choose what pieces of information are worth acquiring and decide how closely to pay attention to them. Filip Matějka and Alisdair McKay (2011) show that the optimal strategy of a rationally inattentive agent faced with a discrete choice problem leads to choice probabilities that follow a generalized multinomial logit model. The generalization is that it is not the true values of the available options that enter the logit formula, but the true values adjusted for the decision maker's prior knowledge and information processing strategy. This close connection to the logit model makes the decision problem tractable and allows us to analyze the equilibrium prices set by firms facing rationally inattentive consumers.

In most models of consumer search the consumer is either not in contact with a given firm or knows that firm's offer perfectly. In our model, the consumer is in contact with all firms but is unsure of the terms

\footnotetext{
* Matějka: CERGE-EI, Politickych veznu 7, Prague 11121, Czech Republic. CERGE-EI is a joint workplace of the Center for Economic Research and Graduate Education, Charles University, and the Economics Institute of the Academy of Sciences of the Czech Republic. filip.matejka@cerge-ei.cz. McKay: Department of Economics, Boston University, 270 Bay State Road, Boston, MA 02215, amckay@bu.edu. We are grateful to Alexander Wolitzky for a helpful discussion. This research was funded by GA ČR P402/11/P236.
}

of their offers. The consumer can decide to evaluate any group of offers he chooses, with any precision, and following any decision tree on how to proceed with the evaluations. Crucially, the consumer faces a cost of acquiring more precise knowledge of the values. Modeling the information friction in this way leads to equilibrium outcomes that differ qualitatively from a standard search model. For example, Diamond (1971) showed that even a small search cost can lead firms to post the monopoly price in equilibrium. This is not true in our model: as the cost of information falls, the equilibrium price falls to the competitive price continuously.

Some of the other findings of our analysis are as follows. First, in a symmetric equilibrium, firms set prices that are increasing in the consumer's cost of processing information and decreasing in the number of firms. Second, when consumers enter the market with prior knowledge of the firms, firms that are viewed favorably by the consumers a priori set higher prices all else equal. Third, adding an offer to the market can increase demand for each of the existing offers. Fourth, when consumers have heterogeneous costs of information, there is a force that leads low-quality firms to set high prices, which is the logic that consumers who do not notice that the quality is low will probably also fail to notice that the price is high. This possibility contrasts with the outcome when consumers are homogeneous, in which case prices are increasing in quality.

Our model is related to work on product differentiation (Simon P. Anderson, Andre de Palma and Jacques-Francois Thisse, 1992), where a logit demand function is derived from a random utility model. Our model differs in the interpretation of the market power enjoyed by firms, but it also has substantive differences in outcomes, 
which stem from the fact that the consumer's prior knowledge of the options affects their choice behavior.

Filip Matějka (2010) has studied the price-setting decision of a single firm facing a rationally inattentive consumer. Here, we consider the equilibrium in a market with firms all facing rationally inattentive consumers. ${ }^{1}$

The paper is organized as follows. In section I we describe the information friction and the consumer's optimal information processing strategy. This section draws heavily on results from Matějka and McKay (2011). In section II we lay out the market environment and then analyze the equilibrium price-setting strategies of firms. We conclude in section III.

\section{The decision problem}

Matějka and McKay (2011) apply the rational inattention framework to a discrete choice setting and relate the resulting choice behavior to the multinomial logit model. Here we summarize the results that are of use in the analysis that follows.

Consider a decision maker who is confronted with a choice among $N$ options with unknown values $v_{1}, \cdots, v_{N}$. The decision maker would like to select the option with the highest value, but gauging the values is costly. From the decision maker's perspective, the values are a random vector $\mathbf{v}$ with distribution $G(\mathbf{v})$. The decision maker can reduce his uncertainty about the realization of $\mathbf{v}$ by paying a cost $\lambda \kappa$ where $\lambda$ is a parameter determining the unit cost of information and $\kappa$ is the amount of information processed as measured by the expected entropy reduction between the decision maker's prior and posterior beliefs about $\mathbf{v} .^{2}$ An information processing strat-

\footnotetext{
${ }^{1}$ Other papers in the rational inattention literature (e.g. Bartosz Mackowiak and Mirko Wiederholt, 2009) have studied the pricing decisions of informationconstrained firms.

${ }^{2}$ If the information processing cost is non-linear, the parameter $\lambda$ would be replaced by a Lagrange multiplier of the dual problem. Under some assumptions, information theory provides foundation for reduction of entropy as the measure of information flow, see Claude E. Shannon (1948).
}

egy can be modeled as a joint distribution between $\mathbf{v}$ and the option selected. A stronger dependence between the choice and $\mathbf{v}$ allows more accurate choices, but also requires processing more information. The decision problem is to choose an information processing strategy so as to maximize the expected value of the selected option less the information processing cost. Matějka and McKay (2011) find that conditional on a realization of $\mathbf{v}$, the probability that the decision maker selects option $i \in\{1, \cdots, N\}$ is

$$
\mathcal{P}_{i}(\mathbf{v}) \equiv \operatorname{Prob}(i \mid \mathbf{v})=\frac{\mathcal{P}_{i}^{0} e^{v_{i} / \lambda}}{\sum_{j=1}^{N} \mathcal{P}_{j}^{0} e^{v_{j} / \lambda}}
$$

The $\left\{\mathcal{P}_{i}^{0}\right\}_{i=1}^{N}$ terms are part of the decision maker's strategy and are equal to the unconditional probability of selecting option $i$ across different realizations of $\mathbf{v}$. Matějka and McKay (2011) show how to solve for these terms. An option that is a priori more attractive will in general have a larger value of $\mathcal{P}_{i}^{0}$ although this is not always the case as, for example, an option that is quite attractive, but always dominated by another will have $\mathcal{P}_{i}^{0}=0$. These terms arise out of the decision maker's prior knowledge and reflect prior beliefs as well as the information processing strategy, which is formed based upon the decision maker's prior knowledge of the options. Therefore, $\left\{\mathcal{P}_{i}^{0}\right\}_{i=1}^{N}$ do not depend on the realization of $\mathbf{v}$. Finally, if the options are exchangeable in the decision maker's prior, i.e. $G(\mathbf{v})$ is invariant to permutations of the entries of $\mathbf{v}$, then $\mathcal{P}_{i}^{0}=1 / N$ for all $i$ and the model reduces to the standard multinomial logit.

\section{Equilibrium prices}

\section{A. The environment}

There are $N$ firms in the market. Firm $i$ is endowed with a technology that produces a good of quality $a_{i}$ at cost $c_{i}$. Let $\theta=\left\{a_{1}, \cdots, a_{N}, c_{1}, \cdots, c_{N}\right\}$ be the vector of exogenous fundamentals. $\theta$ is drawn from a distribution $F(\theta)$.

There is a unit mass of rationally inattentive consumers in the market. Each con- 
sumer will either buy one unit of the good from one of the firms or will choose not to purchase in which case he receives the value $R$, which is the known value of some outside option or reservation value. If the consumer buys from firm $i$, he receives a value of $a_{i}-p_{i}$, where $p_{i}$ is the price set by firm $i$.

We assume that consumers know the distribution of $\theta$ from which costs and qualities are drawn and knows the equilibrium mapping from $\theta$ to prices, but does not know the realization of $\theta$ nor the prices set by the firms. The consumers can refine their prior beliefs about the state of the market by processing information at a cost.

We look for Nash equilibria. Given firm $i$ 's quality $a_{i}$ and cost $c_{i}$, qualities and prices of other firms, $a_{-i}$ and $p_{-i}$, each firm chooses its price to maximize the expected profit:

(2) $\max _{p_{i}} \mathcal{P}_{i}\left(a_{i}-p_{i}, a_{-i}-p_{-i}\right) \times\left(p_{i}-c_{i}\right)$,

where $\mathcal{P}_{i}$ is the expected demand and $\left(p_{i}-\right.$ $\left.c_{i}\right)$ is the firm's markup.

The timing of events is as follows: Nature draws a realization of $\theta$, firms observe $\theta$ and set their prices, consumers process information about the realization of $\theta$ and the prices set by firms and then make their choice. Notice that consumers do not observe the prices set by the firms without paying a cost. In search theory, the cost of observing the price is modeled as the cost of coming into contact with the firm, here it is modeled as the cost of processing information about an offer in hand.

\section{B. General analysis}

Let $p(\theta)$ represent the equilibrium mapping from a realization of firm characteristics to the vector of prices chosen by the firms. ${ }^{3}$ The rationally inattentive consumers are then confronted with a random vector of values, $\mathbf{v}$, with the first $N$ entries given by $a(\theta)-p(\theta)$ and the constant $R$

\footnotetext{
${ }^{3}$ Firms could in general choose to play mixed pricing strategies, but it turns out that they do not. This is due to the demand of the logit-form.
}

as entry $N+1$. The distribution $F(\theta)$ induces a distribution $G(\mathbf{v})$ and this distribution plays the role of the decision maker's prior in the previous section. The consumer forms an information processing strategy including the unconditional choice probabilities $\left\{\mathcal{P}_{i}^{0}\right\}_{i=1}^{N+1}$ and chooses among options in accordance with equation (1). For a given consumer, we can rewrite equation (1) as

$$
\mathcal{P}_{i}=\frac{e^{\left(\alpha_{i}+a_{i}-p_{i}\right) / \lambda}}{\sum_{j=1}^{N} e^{\left(\alpha_{j}+a_{j}-p_{j}\right) / \lambda}+e^{\left(\alpha_{N+1}+R\right) / \lambda}},
$$

where $\alpha_{i}=\lambda \log \left(\mathcal{P}_{i}^{0}\right)$. Writing the choice probabilities this way allows one to see that a firm's unconditional probability of being selected affects its choice of price in the same way that the quality of its product does.

We define an equilibrium in this market as follows.

DEFINITION 1: An equilibrium is a vector of unconditional choice probabilities $\left\{\mathcal{P}_{i}^{0}\right\}_{i=1}^{N+1}$, a pricing function $p(\theta)$, an induced distribution of values $G(\mathbf{v})$ and a conditional choice probability function $\mathcal{P}\left(\mathbf{v} ;\left\{\mathcal{P}_{i}^{0}\right\}_{i=1}^{N+1}\right)$, such that

1) the distribution of fundamentals, $F(\theta)$ and the pricing function induce the distribution of values $G(\mathbf{v})$,

2) $\mathcal{P}\left(\mathbf{v} ;\left\{\mathcal{P}_{i}^{0}\right\}_{i=1}^{N+1}\right)$ and $\left\{\mathcal{P}_{i}^{0}\right\}_{i=1}^{N+1}$ generate a joint distribution between the consumer's choice and $\mathbf{v}$ that solves the consumer's informationconstrained choice problem as described in section $I$,

3) $p_{i}(\theta)$ maximizes the profits of firm $i$ given $\theta, p_{-i}(\theta)$ and the household's choice behavior.

The analysis of an equilibrium is straightforward if one can find the consumer's unconditional choice probabilities, $\left\{\mathcal{P}_{i}^{0}\right\}_{i=1}^{N+1}$, as the consumer's conditional choice probabilities are then given by equation (3) and the pricing function is the solution to a standard logit-demand oligopoly model 
as described in Anderson, de Palma and Thisse (1992).

In the equilibrium of the standard logit model, firms set prices that are increasing in the quality of their products (see Anderson, de Palma and Thisse, 1992). In our model, $\alpha_{i}+a_{i}$ has the same effect as the product quality in the standard logitdemand model. Therefore, firms that are in equilibrium viewed favorably by consumers a priori - in the sense that $\mathcal{P}_{i}^{0}$ and therefore $\alpha_{i}$ are large - also set higher prices.

\section{A priori homogeneous options}

In this subsection, we focus on the case where the consumers do not distinguish between the firms a priori. Consumers only begin to find differences between firms once they start processing information. We also assume there is no outside option (or alternatively, its value approaches $-\infty$ ). Because the firms are exchangeable in the consumer's prior, the consumer selects a strategy such that $\mathcal{P}_{i}^{0}=1 / N$ for all $i$. The model reduces to the logit oligopoly model. Thus, we can apply results from Anderson, de Palma and Thisse (1992) to determine the equilibrium prices. In particular, for a realization of $\theta$ for which the firms are identical, the equilibrium price will be

$$
p=c+\lambda \frac{N}{N-1}
$$

which is increasing in $\lambda$ and decreasing in $N$. The typical interpretation of this result under the random utility derivation of the logit model is that as product differentiation becomes more important to consumers, the equilibrium price rises because competition among the firms is weaker. In our interpretation, competition among the firms becomes weaker as it becomes more costly for consumers to learn about the products on offer. In contrast to the Diamond Paradox, the equilibrium price is continuous in the degree of information friction.

\section{The role of prior knowledge}

When all options are a priori homogeneous, the model behaves just as the stan- dard logit-demand model. When we allow for some a priori heterogeneity in the options, however, the generalized logit model of Matějka and McKay (2011) diverges from the standard logit model and consumer behavior can differ substantially. An easy way to explore the role of prior knowledge is to introduce the no-purchase option. Firms are still assumed to be viewed symmetrically a priori, but they are distinguishable from the reservation option. So from an a priori perspective, the consumer anticipates that he will not purchase with a certain probability, call it $\mathcal{P}^{0}$, and will purchase from any given firm with probability $\left(1-\mathcal{P}^{0}\right) / N$. The demand for the product of firm $i$ is:

$$
\mathcal{P}_{i}=\frac{e^{\left(a_{i}-p_{i}\right) / \lambda}}{\sum_{j=1}^{N} e^{\left(a_{j}-p_{j}\right) / \lambda}+e^{\left(\alpha_{R}+R\right) / \lambda}},
$$

where $\alpha_{R}=\lambda \log \frac{N \mathcal{P}^{0}}{1-\mathcal{P}^{0}}$, which is increasing in $\mathcal{P}^{0}{ }^{4}$

In the standard logit model, i.e. when $\alpha_{R}=0,(1)$ all offers are selected with positive probability, (2) an additional offer (an entrant) decreases demand for the existing offers (at fixed prices), and (3) when one offer is improved, demand for other offers decreases (again at fixed prices).

On the other hand, the choice probabilities following the generalized logit depend on $\alpha_{R}$, which depends on the whole distribution of values relative to the reservation value. For a sufficiently large $R$, the consumer will not process any information and will choose not to purchase from the market. In this case we have $\alpha_{R}=\infty$. Now suppose that one firm changes its strategy to improve its offer or a high-quality firm enters the market. The consumer now views the market more favorably and may now elect to investigate the options, which is to say $\alpha_{R}$ falls. Notice that if the expected value of an offer exceeds $R$ then it is certainly not in the consumer's interest to stay out of the market because even randomly selecting one option yields a higher

\footnotetext{
${ }^{4}$ For a given specification of $F(\theta)$, one can solve for the equilibrium value of $\mathcal{P}^{0}$ using approaches described in Matějka and McKay (2011).
} 
value. Even short of this, an improvement in the offers may induce the consumer to investigate. The resulting reduction in $\alpha_{R}$ benefits all firms and all of their demands go from zero to something positive. Thus, there are two ways that a firm's strategy affects other firms. First, there is the usual market competition channel in which better offers from one firm reduce the demand for other firms, but there is also an information processing channel in which better offers from one firm benefit other firms by prompting the consumer to investigate and purchase.

\section{E. Heterogeneous consumers}

The model can be used to study market equilibria with consumers differing in their costs of processing information. Let there be $K$ groups of consumers with different values of $\lambda$. Each group has size $\gamma_{k}$. We will continue to assume that the firms enter the consumers' priors symmetrically and we again omit the outside option.

For a given realization of $\theta$, let $\mathcal{P}_{i}^{k}$ be the market share of firm $i$ among consumers with information cost $\lambda_{k}$. The first order condition for the firm's choice of price yields ${ }^{5}$

$$
p_{i}=c_{i}+\frac{\sum_{k} \gamma_{k} \mathcal{P}_{i}^{k}}{\sum_{k} \gamma_{k} \mathcal{P}_{i}^{k}\left(1-\mathcal{P}_{i}^{k}\right) / \lambda_{k}} .
$$

If the realization of $\theta$ is such that the firms are identical, we have a symmetric equilibrium with price

$$
p=c+\frac{N}{N-1}\left[E\left(\lambda_{k}^{-1}\right)\right]^{-1},
$$

where the expectation is taken over the $\lambda_{k} \cdot{ }^{6}$ Notice that by Jensen's inequality the equilibrium price for heterogeneous consumers is lower than the one that would arise if all

\footnotetext{
${ }^{5}$ Paul Milgrom and John Roberts (1990) show that the logit oligoply model has a unique equilibrium. Their argument can be extended to cover the case of heterogeneous consumers.

${ }^{6}$ The derivation of this result follows the same steps as the derivation of the analogous result for homogeneous consumers, which can be found in Anderson, de Palma and Thisse (1992).
}

consumers were homogeneous with the average cost of information.

When the firms are also heterogeneous, the model behaves qualitatively differently. The homogeneous-consumer model predicts that prices are increasing in quality (see Anderson, de Palma and Thisse, 1992). However, when consumers are heterogeneous, prices can be decreasing in quality. We demonstrate this type of result in Figure $1 .^{7}$ The intuition is that firms want to set a markup that is related to the information processing costs of the consumers to whom they sell. Low-quality firms expect to sell to consumers with high costs of information and as a result would like to set a large markup. The figure also shows the homogeneous-consumer case and here one can see that the equilibrium price is higher for all levels of quality and is increasing in quality.

The baseline homogeneous-consumer model generates the same probabilistic demand and market equilibria as the random utility model with tastes that are extreme value distributed. In this case, prices always increase with quality. On the other hand, the heterogeneous-consumer model corresponds to a random utility model with tastes distributed according to a mixture of different extreme value distributions, where we now know prices can decrease with quality. This casts some doubts on the robustness of results derived from random utilities as seemingly minor changes to the taste distributions can drastically change the resulting market equilibria.

\footnotetext{
${ }^{7}$ To solve for the equilibrium prices, we guess a vector of prices and then use an iterative algorithm based on equation (4).
} 


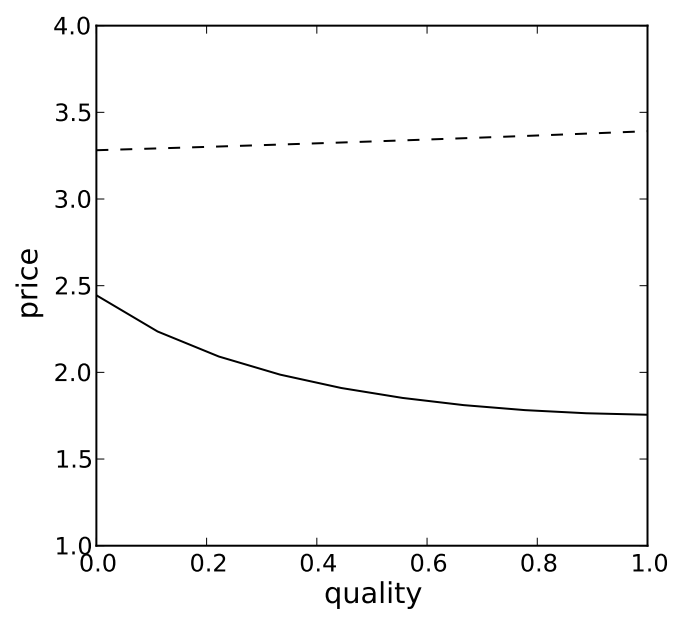

Figure 1. Equilibrium PRICES AMONG 10 Firms With QUalities EQUally SPACED Between 0 AND 1 . Solid LINE: MARKET HAS TWO EQUALLY-SIZED GROUPS OF CONSUmers with $\lambda=1$ AND $\lambda=5$. DAShed Line: HOMOGeneOUs CONSUMERS With $\lambda=3$.

\section{Conclusion}

We have studied equilibria in markets where consumers find it costly to process information about existing offers and shown that this model generates equilibria robust to small deviations in information cost, unlike most sequential search models. The market equilibria are in some cases equivalent to equilibria generated by the standard logit-demand model and in some other cases they differ significantly. While some have argued that the rational inattention framework leads to problems that are intrinsically too complex, the model itself is surprisingly simple to solve. This provides an array of possible future applications. For instance, there is a natural connection between our model and the literature on obfuscation (e.g. Glenn Ellison and Alexander Wolitzky, 2011), which aims to understand practices by firms that serve to make the terms of their offers less transparent.

\section{REFERENCES}

Anderson, Simon P., Andre de Palma, and Jacques-Francois Thisse. 1992. Discrete Choice Theory of Product Differentiation. Cambridge:MIT Press.
Burdett, Kenneth, and Kenneth L. Judd. 1983. "Equilibrium Price Dispersion." Econometrica, 51(4): 955-69.

Diamond, Peter A. 1971. "A model of price adjustment." Journal of Economic Theory, 3(2): 156-168.

Ellison, Glenn, and Alexander Wolitzky. 2011. "A Search Cost Model of Obfuscation." MIT Working Paper.

Mackowiak, Bartosz, and Mirko Wiederholt. 2009. "Optimal Sticky Prices under Rational Inattention." The American Economic Review, 99.

Matějka, Filip. 2010. "Rigid Pricing and Rationally Inattentive Consumer." CERGE-EI Working Papers wp409.

Matějka, Filip, and Alisdair McKay. 2011. "Rational Inattention to Discrete Choices: A New Foundation for the Multinomial Logit Model." Boston University Working Paper.

Milgrom, Paul, and John Roberts. 1990. "Rationalizability, Learning, and Equilibrium in Games with Strategic Complementarities." Econometrica, 58(6): 1255-77.

Shannon, Claude E. 1948. "A Mathematical Theory of Communication." The Bell System Technical Journal, 27.

Sims, Christopher A. 2003. "Implications of Rational Inattention." Journal of Monetary Economics, 50(3).

Sims, Christopher A. 2006. "Rational Inattention: Beyond the LinearQuadratic Case." The American Economic Review, 96(2). 